\title{
Synthesis and Characterization of Magnetic Colloids
}

\author{
S. Savitha and Sarbari Bhattacharya*
}

Magnetic fluids which constitute a suspension of magnetic nanoparticles in a carrier liquid are termed ferrofluids. Ferrofluids are smart fluids exhibiting both magnetic and fluid like characteristics. In the present study, we report on ferrofluidswhich are suspensions of magnetite $\left(\mathrm{Fe}_{3} \mathrm{O}_{4}\right)$ nanoparticles in carrier fluids of varying viscosity. The magnetite nanoparticles have been synthesized by a chemical co-precipitation method.X-Raydiffraction of the as prepared sample revealsa single phase of magnetite.SEM characterisation reveals regular morphology and a narrow size distribution for the synthesized nanoparticles. These are dispersed in pure water and mixtures of water and glycerol using suitable surfactants and ultrasonicated. Our studies reveal that the stability of the ferrofluids have a strong dependence on the viscosity of the carrier fluid as well as the $\mathrm{pH}$, with high viscosity and low $\mathrm{pH}$ yielding more stable ferrofluids. While low concentration of the magnetic component renders it impossible to see signatures of its presence in Raman spectroscopy, SQUID magnetometryreveals the presence of the magnetic component suggesting a distribution in size of the nanoparticles with a broad blocking temperature range. Further, a magnetic anomaly is seen at the freezing point of the carrier fluid in the ZFC-FC measurements for the ferrofluid made with $50 \% \mathrm{v} / \mathrm{v}$ aqueous glycerol solution.

microfluidic flow.Ferrofluids have enormous potential application in drug delivery, hyperthermia and MRI contrast agents. ${ }^{[3]}$

Ferrofluids differ from magnetorheological (MR) fluids in terms of the size of the particles that form the magnetic component which in turn influences their stability. Magnetic particles in MR fluids, being micron sized invariably settle over time under the influence of gravity. True ferrofluids are supposed to be always stable. However, even in ferrofluids, breakdown of surfactants over time results in agglomeration of nanoparticles, which in turn affects both the magnetic response as well as the stabilitiy of ferrofluids.

In the work presented here, magnetite $\left(\mathrm{Fe}_{3} \mathrm{O}_{4}\right)$ particles are synthesized by coprecipitation $^{[4]}$ method and are characterized by X-Ray Diffraction (XRD) and Scanning Electron Microscope (SEM). Ferrofluids are prepared by dispersing as prepared magnetite in pure water and water-glycerol mixture with the help of a suitable surfactant. An increase in the viscosity of the carrier fluid is proposed to be a way of improving the stability of a

\section{Introduction}

Ferrofluids are stable colloidal fluids that respond strongly to a magnetic field. They are usually made by suspending magnetic nanoparticles in a carrier fluid. The nanoparticles are usually coated with surfactants to inhibit their aggregation.Ferrofluids do not retain their magnetic properties on removal of magnetic field. This is possible because nanoparticles involved are superparamagnetic in nature. Ferrofluids are interesting smart fluids as they exhibit both magnetic and fluid like characteristics and are of great research interest because of their wide spreadapplications in the field of biomedicine and engineering. Since the synthesis of the first stable colloidal ferrofluid in $1938^{[1]}$ and the commercial use of ferrofluids by NASA to control motion of fluids in space in $1960,{ }^{[2]}$ ferrofluids have been incorporated in a number of industrial applications like sealing, transformer cooling, dampening and cooling agents in loud speakers, recovery of hazardous wastes and controlled

S. Savitha, S. Bhattacharya

Department of Physics Bangalore University, Bangalore-560056,

Karnataka, India

E-mail: sarbari.bhattacharya@bub.ernet.in

DOI: 10.1002/masy.201600195 ferrofluid. Apart from studying and comparing the stability of these ferrofluids, we have tried to understand the effect of changed carrier fluid viscosity on the properties of the ferrofluids.The ferrofluids synthesized, have therefore been studied for their magnetic behavior and chemical structural properties through Superconducting Quantum Interference Device (SQUID) and Raman spectroscopy.

\section{Experimental Section}

\subsection{Materials}

Materials used for synthesis of ferrofluid are $\mathrm{FeCl}_{3} \cdot 6 \mathrm{H}_{2} \mathrm{O}, \mathrm{FeSO}_{4} \cdot 7 \mathrm{H}_{2} \mathrm{O}$, $\mathrm{NaOH}, \mathrm{HCl}$, Tetra Methyl Ammonium Hydroxide (TMAOH), pure water and glycerol. Reagent grade materials were used without further purification. Distilled water was used as a solvent.

\subsection{Methods}

\subsubsection{Synthesis of Magnetite}

The method of synthesis followed for $\mathrm{Fe}_{3} \mathrm{O}_{4}$ nanoparticles is as described in Ref. [4] The preparedsolution is washed several 
Table 1. Physical properties of carrier fluids. ${ }^{[7]}$

\begin{tabular}{lcc}
\hline Carrier fluid denisty $\left(10^{3} \mathrm{~kg} / \mathrm{m}^{3}\right)$ & Viscosity $\left(30^{\circ} \mathrm{C}\right)(\mathrm{mPa} . \mathrm{s})$ & Freezing point $\left({ }^{0} \mathrm{~K}\right)$ \\
\hline Water 0.99567 & 1.002 & 273 \\
Water-Glycerol $1.12096(50 \% \mathrm{v} / \mathrm{v})$ & 4.21 & 251.6 \\
\hline
\end{tabular}

times with distilled water till $\mathrm{pH}$ is between 9 and 10. After removing excess water the wet precipitate is dried in furnace at $150^{\circ} \mathrm{C}$ for $3 \mathrm{~h}$. The resulting powder is used for XRD and SEM characterization.

The carrier fluid is the dominant component and governs the physical properties of the ferrofluid. The carrier fluid is usually an organic solvent/hydrocarbon/inorganic solvent. ${ }^{[5]}$ Water based ferrofluids are important for biomedical applications. Glycerol $\left(\mathrm{C}_{3} \mathrm{H}_{5}(\mathrm{OH})_{3}\right)$ is a clear, odorless, viscous, stable, nontoxic and widely compatible liquid. Water and glycerol are said to be miscible in any proportion. The viscosity of aqueous glycerol solution can be varied in continuous manner from 0.8007 Centipoise for pure water to 612 Centipoise for pure glycerol at room temperature by using different proportions of the two components. ${ }^{[7]}$ TMAOH is used as surfactant in the present work.It is a cationic surfactant i.e., its head group is positively charged. ${ }^{[6]}$ Thus positive charges are attached to magnetite particles resulting in an electrical repulsion between them and preventing their agglomeration. Given below is Table 1 showing the density, viscosity and freezing point of pure water and an aqueous glycerol solution with equal proportions of water and glycerol. ${ }^{[7]}$

\subsubsection{Synthesis of Ferrofluid}

Magnetite powder is soaked overnight in $1 \mathrm{M} \mathrm{NaOH}$ solution and then dried in oven at $70^{\circ} \mathrm{C}$ for $2 \mathrm{~h}$. The powder is washed with hot water several times to remove $\mathrm{Na}$ particles on the surface. ${ }^{[8]}$ The wet particles are dispersed in pure water and water-glycerol $(50 \% \mathrm{v} / \mathrm{v})$ along with $10 \mathrm{v} \%$ of surfactant and ultrasonicatedeither using a bath or probe till the nanoparticles are completely dispersed in liquid. While about 90 minutes were required for the ultrasonication in the case of the bath only 15 minutes were sufficient for the probe. Care was taken to ensure that there was no heating of sample during the sonication process.

Three samples were prepared and labelled as s1, s2 and s3. The first sample was the as prepared magnetite in powder form (s1), the second one, magnetite dispersed in pure water with TMAOH as surfactant (s2) while the third sample was magnetite powder dispersed in water-glycerol mixture $(50 \mathrm{v} / \mathrm{v} \%)$ with TMAOH as surfactant (s3). In the cases of s2 and s3, $1 \mathrm{ml}$ of the as prepared wet nanoparticles was dispersed in $50 \mathrm{ml}$ of carrier fluid.

The powder X-Ray Diffraction measurements of sample s1 was carried out by an X-ray diffractometer (RigakuUltima IV) with $\mathrm{CuKa}$ radiation. The Raman Spectra were recorded using the $532 \mathrm{~nm}$ excitation line in a VENTANA Raman spectrometer. The magnetic properties of the as prepared magnetite as well as the ferrofluid synthesized were measured with SQUID (Quantum Design) magnetometer with a capacity to use magnetic fields up to $7 \mathrm{~T}$.

\section{Results and Discussions}

\subsection{Structural and Morphological Analysis}

The X-ray diffraction of sample s1 is as shown in Figure 1(a). The prepared sample appears to be in single phase and peaks match with that of the standard pattern for $\mathrm{Fe}_{3} \mathrm{O}_{4}$ (JCPDS 190629). The average crystalline size is calculated using Debye Scherrer's relation, $D=0.9 \lambda / \beta \cos \theta$, where $\lambda$ is the $\mathrm{X}$-ray wavelength $\left(1.5406 \mathrm{~A}^{0}\right.$ for $\left.\mathrm{CuK \alpha}\right)$ and $\beta$ is the full width at half maximum (FWHM) in radians calculated using Gaussian fitting. ${ }^{[9,10]}$ Taking the peak with the highest intensity, here for (311), $\theta$ is noted and the particle size is estimated. The estimated size is found to be $13 \mathrm{~nm}$. The magnetite nanoparticle morphology was investigated by SEM. A typical

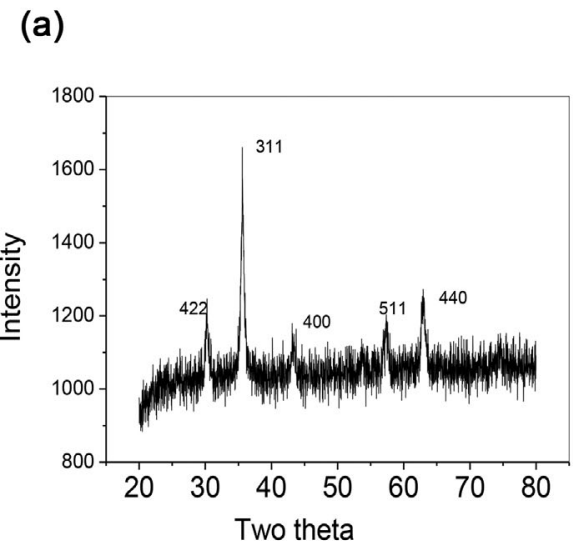

(b)

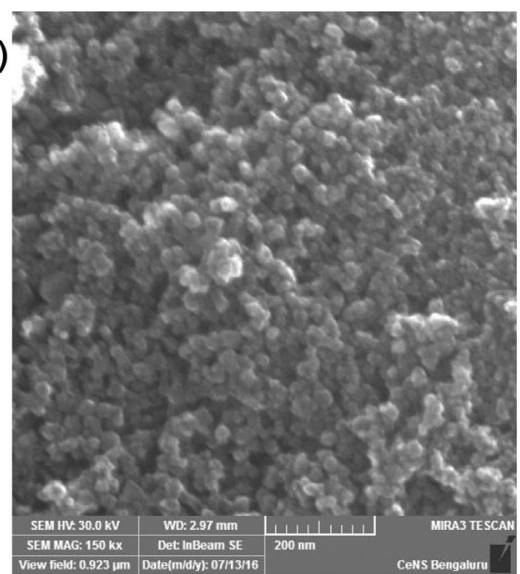

Figure 1. a) XRD pattern of as prepared sample (s1) revealing single phase of magnetite (b) SEM image of as prepared sample (s1). 


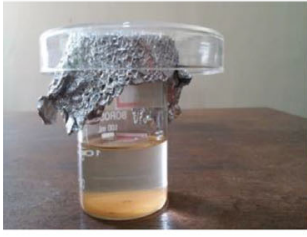

(a)

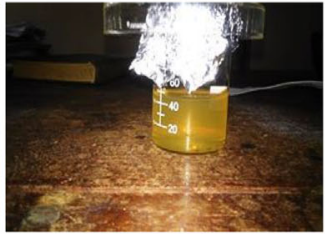

(b)

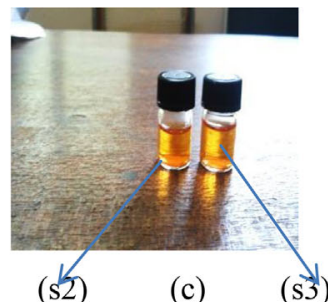

(c)

(s)

Figure 2. a) Ferrofluid with low viscosity carrier fluid and high $\mathrm{pH}$ (unstable beyond 10 hours) (b) ferrofluid with high carrier fluid viscosity and high $\mathrm{pH}$ (unstable beyond three days) (c) stable ferrofluid low carrier fluid viscosity and low pH (s2) and high carrier fluid viscosity and low pH (s3) (stable up to a week).

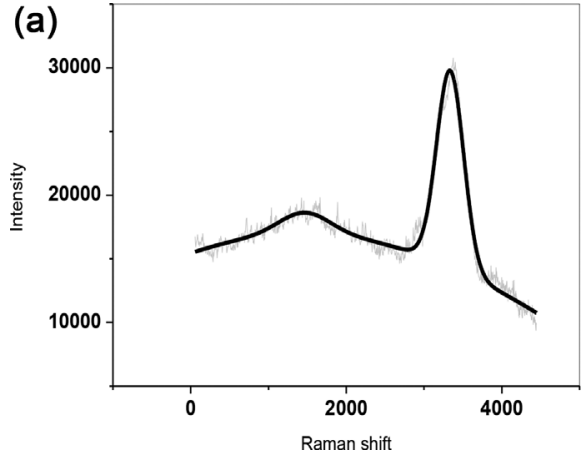

Figure 3. Raman spectra of ferrofluids (a) sample s2 (b) sample s3.

SEM image is shown in Figure 1(b). As can be seen, the sample consists of particles with nearly spherical shape and extremely narrow size distribution.

\subsection{Stability Studies}

There are many competing forces between nanoparticles in a ferrofluid such as inter grain interactions due to attractive Van der Waal, dipole-dipole forces and repulsive electrostatic or steric repulsive forces due to charge on the nanoparticles. Steric repulsion is produced due to coating with an appropriate surfactant. $^{[11]}$ Stability of ferrofluid can be increased by increasing the viscosity which works by increasing Brownian relaxation time of nanoparticles. Stability factor also depends on $\mathrm{pH}$ of the solution. Highly stable fluids can be obtained by having a ferrofluid of $\mathrm{pH}$ factor equal to $7 .^{[12]}$ We find on studying the undisturbed ferrofluid over time that sample s3 is much more stable than sample s2. While phase separation and setting under gravity was evident in low viscosity and high $\mathrm{pH}$ ferrofluid in a few hours of time, no visible change was evident in s3 after a couple of weeks (Figure 2).

\subsection{Raman Characterization}

The samples (s2 and s3) were loaded into glass cuvettes for the measurement process. The well-defined peaks at 3357, 2881 and

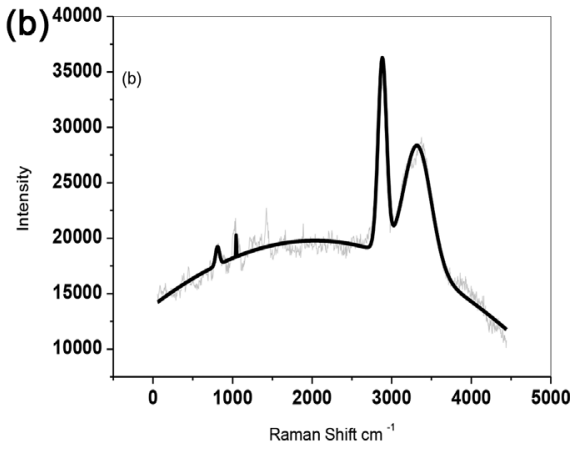

$3341 \mathrm{~cm}^{-1}$ are associated with molecular vibration of the carrier fluids. The vibrational frequencies associated with hydrogen motion in liquid water lead to a broad peak at $3400 \mathrm{~cm}^{-1}$ and are due to O-H stretching mode, ${ }^{[13]}$ whereas the glycerol contribution corresponds to well defined peak at $2881 \mathrm{~cm}^{-1}$. The intensity of Raman peaks associated with the carrier fluids is high masking all possible contribution from the magnetite nanoparticles. This is because the nanoparticle concentration is very low in these ferrofluids (Figure 3).

\subsection{Magnetic Characterizations}

The magnetization measurement was from -30 to $+30 \mathrm{kOe}$ field. From the variation of magnetization with the applied field

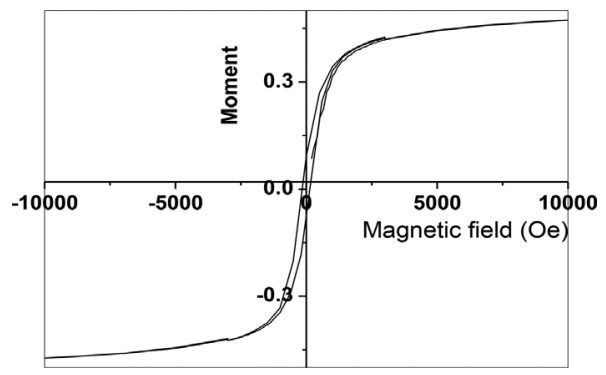

Figure 4. Magnetization curve of $\mathrm{s} 1$ (at $50 \mathrm{~K}$ ). 

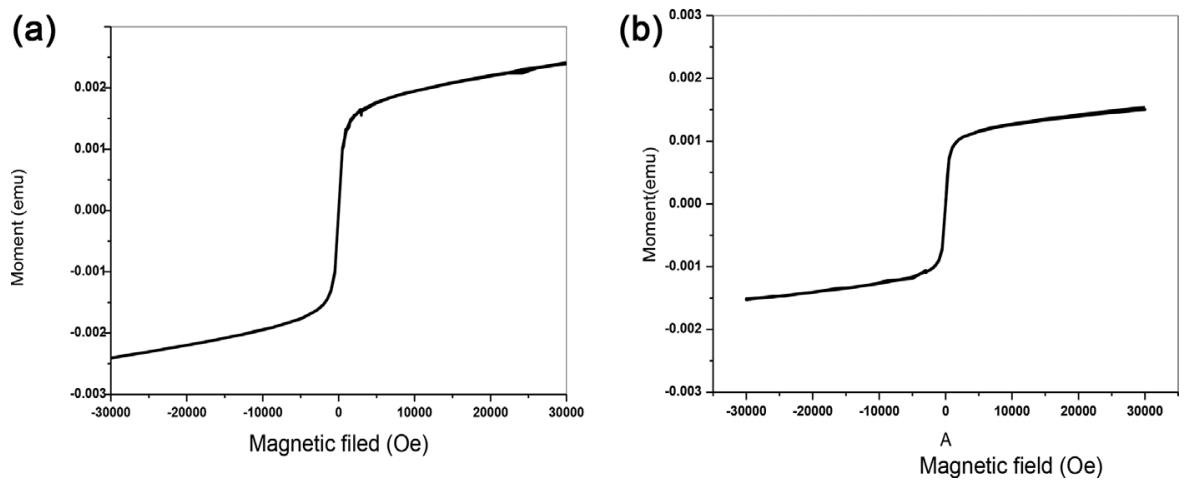

Figure 5. a) Magnetization curve of s2. b) Magnetization curve of s3.
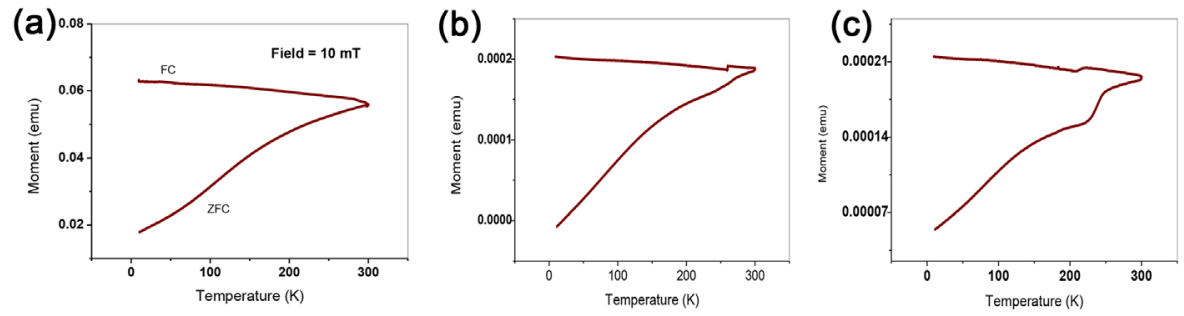

Figure 6. a) FC/ZFC curves of sample s1 (b) sample s2 (c) sample s3.

at $50 \mathrm{~K}$, the saturation magnetization $(\mathrm{Ms})$ of magnetite was measured to be $74 \mathrm{emu} / \mathrm{g}\left(74 \times 10^{3} \mathrm{~A} / \mathrm{m}\right)$. The magnetization curve with hysteresis loop having a coercivity of $25 \mathrm{mT}$, is shown in Figure 4, confirming ferrimagnetic nature.

Magnetization curves shown in Figure 5(a) and (b) at RT do not show any hysteresis loop with measurable values of coercivity and retentivity. This confirms that the synthesized samples exhibit superparamagnetism at RT. From the magnetization curves (a) and (b) it appears that as the viscosity increases, magnetization decreases. ${ }^{[10]}$

Figure 6 shows FC-ZFC dc magnetization curves for samples s1, s2 and s3 measured using a magnetic field of $10 \mathrm{mT}$. The ZFC shows a broad blocking behavior at $200 \mathrm{~K}$ with a maximum magnetic moment at $300 \mathrm{~K}$. The blocking temperature is defined as the temperature at which nanoparticles do not relax during the time of measurement. Each nanoparticle is blocked along its easy magnetization axis and this behavior depends on particle anisotropy, volume and orientation. The broad blocking behavior indicates a distribution of particle volume and hence each particle is blocked at different $\mathrm{T}_{\mathrm{B}}$.

For sample s3, a magnetic anomaly about $250 \mathrm{~K}$ is observed. Freezing temperature of water-glycerol, $250 \mathrm{~K}$, is above $\mathrm{T}_{\mathrm{B}}$, which is $200 \mathrm{~K}$. As freezing and blocking are both contributing to the physical motion of nanoparticles and alignment of movement in the field direction, the cusp is more pronounced compared to other graphs. ${ }^{[14]}$ Further the saturation of magnetic moment at low temperature in FC curve indicates a strong interaction between nanoparticles. ${ }^{[15]}$

\section{Conclusion and Future work}

We have studied ferrofluids prepared from magnetite nanoparticles synthesized using co-precipation method and suspended in carrier fluids of pure water and a $50 \% \mathrm{v} / \mathrm{v}$ aqueous glycerol solution. We find that the stability of the ferrofluids have a strong dependence on the viscosity of the carrier fluid as well as the $\mathrm{pH}$. Raman spectroscopy was unable to give insights into the possible chemical structure alterations of the magnetic particles in these ferrofluids owing to the low concentration of magnetic nanoparticles. SQUID magnetometry measurements on the as prepared magnetic nanoparticles show an appreciable saturation magnetization comparable to that reported in literature. ${ }^{[16]}$ Room temperature measurements reveal that higher carrier fluid viscosity ferrofluids show lowered saturation magnetization. The ZFC-FC measurements also reveal a magnetic anomaly for the ferrofluid made using a carrier fluid of $50 \% \mathrm{v} / \mathrm{v}$ aqueous glycerol solution at a temperature which can be associated with the freezing of the carrier fluid. Further systematic studies using aqueous glycerol solutions with different proportions of water and glycerol as carrier fluids will give us insight into the effect of altered carrier fluid viscosity on the stability and magnetic properties of the ferrofluid.

\section{Acknowledgements}

The authors wish to acknowledge UGC, DST PURSE and Department of Biotechnology, Bangalore University. 


\section{Keywords}

carrier fluids, co-precipitation, ferrofluid, surfactants, viscosity

[1] S. May, "The rotational relaxation dynamics of ferrofluid system", Master Thesis.

[2] S. M. Hakim, "Synthesis and Characterization of ferrofluid from Iron and Manganese", Master Thesis, 2012.

[3] B. Lee, preserve@lehigh.edu, 2007, Vol 15-2007, paper 5.

[4] P. Berger, N. B. Adelman, K. J. Beckman, D. J. Campbell, A. B. Ellis, G. C. Lisensky, J. Chem. Edu. 1999, 76, 943.

[5] K. Raj, B. Moskowitz, R. Casciari, J. Magnetism Magnetic Mater. 1995, 149, 174.

[6] S. Scherer, A. M. FigueiredoNeto, Brazillian J. Phys. 2005, 35.

[7] Physical Properties of Glycerine and Its Solutions. Gulf Publishing Company, 1957.

[8] S. S. Pati, V. Mahendran, J. Philip, J. Nanofluids 2013, 2, 94.
[9] Priyanka, Simran, A. Singh, V. Sharma, Int.J. Eng. Res. Technol. 2013,

[10] M. C. Mascolo, Y. Pei, T. A. Ring, Materials 2013, 6, 5549.

[11] R V. Mehta, R. V. Upadhyay, Curr. Sci. 1999, 76, 305.

[12] A. S. Drozdov, V. Ivanovski, D. Avnir, V. V. Vinogradov, J. Colloid Interface Sci. 2016, 468, 307.

[13] J. E. Weber, A. R. Goni, D. J. Pusiol, C. Thomsen, Phys. Rev. E 2002 66, 021407

[14] M. B. Morales, M. H. Phan, S. Pal, N. A. Frey, H. Sikanth, J. Appl. Phys. 2009, 105, 07b511.

[15] M. Marolt, superparamagnetic materials, seminar content, 2014

[16] A. P. Reena Mary, T. N. Narayanan, V. Sunny, D. Sakthikumar, Y. Yoshida, P. A. Joy, M. R. Anantharaman, Nanoscale Res. Lett. 2010, 5, 1706.

[17] J. A. Lopez, F. Gonzalez, F. A. Bonilla, G. Zamrano, M. E. Gomez, Revista Latinoamericana de MetallurgiaY Materials 2010, 30, 60 .

[18] L. F Gamarra, W. M. Pontuschka, J. B. Mamani, D. R. Cornejo, T. R. Oliveira, E. D. Vieira, A. J. Costa-Filho, E. aro, Jr., J. Phys. Condens. Matter 2009, 21, 115104. 